\title{
Stress-Energy-Momentum Tensors and the Belinfante-Rosenfeld Formula
}

\author{
Mark J. Gotay* \\ Mathematics Department \\ U.S. Naval Academy, Annapolis, MD 21402-5002 \\ Jerrold E. Marsden ${ }^{\dagger}$ \\ Department of Mathematics \\ University of California, Berkeley, CA 94720
}

17 April 2001

\begin{abstract}
We present a new method of constructing a stress-energy-momentum tensor for a classical field theory based on covariance considerations and Noether theory. The stress-energy-momentum tensor $\mathcal{T}^{\mu}{ }_{\nu}$ that we construct is defined using the (multi)momentum map associated to the spacetime diffeomorphism group. The tensor $\mathcal{T}^{\mu}{ }_{\nu}$ is uniquely determined as well as gauge-covariant, and depends only upon the divergence equivalence class of the Lagrangian. It satisfies a generalized version of the classical Belinfante-Rosenfeld formula, and hence naturally incorporates both the canonical stress-energy-momentum tensor and the "correction terms" that are necessary to make the latter well behaved. Furthermore, in the presence of a metric on spacetime, our $\mathcal{T}^{\mu \nu}$ coincides with the Hilbert tensor and hence is automatically symmetric.
\end{abstract}

\section{Introduction}

For many years classical field theorists have grappled with the problem of constructing a suitable stress-energy-momentum ("SEM") tensor for a given collection of fields. There are various candidates for this object; for instance, from spacetime translations via Noether's theorem one can build the so-called canonical SEM tensor density

$$
\mathfrak{t}^{\mu}{ }_{\nu}=L \delta^{\mu}{ }_{\nu}-\frac{\partial L}{\partial \phi_{, \mu}^{A}} \phi_{, \nu}^{A}
$$

\footnotetext{
* Research partially supported by NSF Grant DMS 88-05699

${ }^{\dagger}$ Research partially supported by NSF Grant DMS 89-22704
} 
where $L$ is the field Lagrangian, $\mu$ and $\nu$ are spacetime indices, and $\phi^{A}$ are the field components. Unfortunately, $\mathfrak{t}^{\mu \nu}$ is typically neither symmetric nor gauge-invariant, and much work has gone into efforts to "repair" it, cf. Belinfante [1939], Wentzel [1949], Corson [1953] and Davis [1970].

General relativity provides an entirely different method of generating a SEM tensor (Hawking and Ellis [1973], Misner, Thorne and Wheeler [1973]). For a matter field coupled to gravity,

$$
\mathfrak{T}^{\mu \nu}=2 \frac{\delta L}{\delta g_{\mu \nu}}
$$

defines the Hilbert SEM tensor density. By its very definition, $\mathfrak{T}^{\mu \nu}$ is both symmetric and gauge-covariant. Despite its lack of an immediate physical interpretation, this "modern" construction of the SEM tensor has largely supplanted that based on Noether's theorem. Formulae like this are also important in continuum mechanics, relativistic or not. For example, in nonrelativistic elasticity theory, (1.2) is sometimes called the Doyle-Eriksen formula and it defines the Cauchy stress tensor. This formula has been connected to the covariance of the theory by Marsden and Hughes [1983] and Simo and Marsden [1984].

Discussions and other definitions of SEM tensors and related objects can be found in Souriau [1974], Kijowski and Tulczyjew [1978] as well as Ferraris and Francaviglia [1985, 1991]. In general, however, the physical significance of these proposed SEM tensors remains unclear. In field theories on a Minkowski background, $\mathfrak{t}^{\mu \nu}$ is often symmetrized by adding to it a certain expression which is attributed to the energy density, momentum density, and stress arising from spin. While one can give a definite prescription for carrying out this symmetrization (Belinfante [1939]), such modifications are nonetheless ad hoc. The situation is more problematical for field theories on a curved background, or for "topological" field theories in which there is no metric at all. Even for systems coupled to gravity, the definition (1.2) of $\mathfrak{T}^{\mu \nu}$ has no direct physical significance. Now, the Hilbert tensor can be regarded as a constitutive tensor for the matter fields by virtue of the fact that it acts as the source of Einstein's equations. But this interpretation of $\mathfrak{T}^{\mu \nu}$ is in a sense secondary, and it would be preferable to have its justification as a SEM tensor follow from first principles, i.e., from an analysis based on symmetries and Noether theory.

Moreover the relations between various SEM tensors, and in particular the canonical and Hilbert tensors, is somewhat obscure. On occasion, $\mathfrak{T}^{\mu \nu}$ is obtained by directly symmetrizing $\mathfrak{t}^{\mu \nu}$ (as in the case of the Dirac field), but more often not (e.g., electromagnetism). For tensor or spinor field theories, Belinfante [1940] and Rosenfeld [1940] (see also Trautman [1965]) showed that $\mathfrak{T}^{\mu}{ }_{\nu}$ can be viewed as the result of "correcting" $\mathfrak{t}^{\mu}{ }_{\nu}$ :

$$
\mathfrak{T}^{\mu}{ }_{\nu}=\mathfrak{t}^{\mu}{ }_{\nu}+\nabla_{\rho} K_{\nu}^{\mu \rho}
$$

for some quantities $K_{\nu}^{\mu \rho}$. We refer to (1.3) as the Belinfante-Rosenfeld formula.

Our purpose in this paper is to give a physically meaningful definition of the SEM tensor based on covariance considerations for (essentially) arbitrary field theories 
that suffers none of these maladies. We will show that the SEM tensor density so defined (we call it $\mathcal{T}^{\mu}{ }_{\nu}$ ) satisfies a generalized version of the Belinfante-Rosenfeld formula (1.3), and hence naturally incorporates both $\mathfrak{t}^{\mu}{ }_{\nu}$ and the "correction terms" which are necessary to make the latter gauge-covariant. Furthermore, in the presence of a metric on spacetime, we will show that our SEM tensor coincides with the Hilbert tensor, and hence is automatically symmetric.

The key ingredient in our analysis is the (multi)momentum map associated to the spacetime diffeomorphism group. Using this object we define the SEM tensor density $\mathcal{T}^{\mu}{ }_{\nu}$ by means of fluxes of the multimomentum map across hypersurfaces in spacetime. This makes intuitive sense, since the multimomentum map describes how the fields "respond" to spacetime deformations. One main consequence is that our definition uniquely determines $\mathcal{T}^{\mu}{ }_{\nu}$; this is because our definition is "integral" (i.e., in terms of fluxes) as opposed to being based on differential conservation laws as is traditionally done, $c f$. Davis [1970]. Thus unlike, say, $\mathfrak{t}^{\mu}{ }_{\nu}$, our SEM tensor is not merely defined up to a curl, and correspondingly there is no possibility of - and no necessity for - modifying it. The fact that the relevant group is the entire spacetime diffeomorphism group, and not just the translation group, is also crucial. Indeed, nonconstant deformations are what give rise to the "correction terms" mentioned above. Moreover, our analysis is then applicable to field theories on arbitrary spacetimes (in which context of course the translation group, let alone the Poincaré group, no longer has global meaning).

The plan of the paper is the following. In $\S 2$ we supply some of the relevant background on Lagrangian field theory and multimomentum maps. In $\S \mathbf{3}$ we define the SEM tensor $\mathcal{T}^{\mu}{ }_{\nu}$ and derive a local expression for it, in the process establishing generalized versions of the Belinfante-Rosenfeld and Hilbert formulas (1.3) and (1.2). Here we also prove that $\mathcal{T}^{\mu}{ }_{\nu}$ so defined is gauge-covariant and depends only upon the divergence equivalence class of the Lagrangian. In $\S 4$ we discuss in detail the important special case of a field theory coupled to either a metric or a frame on spacetime. We present several examples of our results in $\S \mathbf{5}$.

\section{Lagrangian Field Theories}

Here we rapidly sketch those aspects of Lagrangian field theory that we need for our development. ${ }^{1}$ We adopt the spacetime covariant approach of Gotay et al. [1992], to which we refer the reader for further details. We restrict attention to first order theories, although we expect that the results obtained here can be extended to higher order theories.

Let $X$ be an $(n+1)$-dimensional parameter space, which in applications is usually, but not always, spacetime, and let $\pi_{X Y}: Y \rightarrow X$ be a bundle whose sections are the fields of interest. The field theoretic analogue of the tangent bundle of mechanics is the first jet bundle $J^{1} Y$. The Lagrangian density is a bundle map $\mathcal{L}: J^{1} Y \rightarrow \Lambda^{n+1} X$ over $X$, where $\Lambda^{n+1} X$ is the bundle of $(n+1)$-forms on $X$.

\footnotetext{
${ }^{1}$ We shall work exclusively in the Lagrangian picture. For a discussion of the covariant Hamiltonian approach to field theory, see Gotay et al. [1992].
} 
Denote the corresponding Cartan form by $\Theta_{\mathcal{L}}$; it is an $(n+1)$-form on $J^{1} Y$.

Let $\mathcal{G}$ be the gauge group of the theory, realized as a subgroup of $\operatorname{Aut}(Y)$. We shall not give a precise definition of $\mathcal{G}$ here, but simply regard it as given. We assume that the Lagrangian field theory under consideration is parametrized in the sense that the image of $\mathcal{G}$ under the natural projection $\operatorname{Aut}(Y) \rightarrow \operatorname{Diff}(X)$ contains the group $\operatorname{Diff}_{c}(X)$ of compactly supported diffeomorphisms. ${ }^{2}$ For a discussion of parametrized theories, see Kuchař [1973]..$^{3}$

Our fundamental assumption is that $\mathcal{L}$ is $\mathcal{G}$-equivariant, which means that

$$
\mathcal{L}\left(\eta_{J^{1}(Y)}(\gamma)\right)=\left(\eta_{X}\right)_{*} \mathcal{L}(\gamma)
$$

for all $\gamma \in J^{1} Y$ and all $\eta_{Y} \in \mathcal{G}$, where $\eta_{J^{1}(Y)}$ is the prolongation of the automorphism $\eta_{Y}$ of $Y$ to $J^{1} Y$ and $\eta_{X}$ is the induced diffeomorphism of $X$. From this it follows that $\Theta_{\mathcal{L}}$ is $\mathcal{G}$-invariant, in which case we say that the field theory is $\mathcal{G}$-covariant. Associated to such an action is a canonical covariant or multimomentum map $J^{\mathcal{L}}: J^{1} Y \rightarrow \mathfrak{g}^{*} \otimes \Lambda^{n}\left(J^{1} Y\right)$ given by

$$
\left\langle J^{\mathcal{L}}(\gamma), \xi_{Y}\right\rangle=\mathbf{i}\left(\xi_{J^{1} Y}(\gamma)\right) \Theta_{\mathcal{L}}
$$

where $\mathfrak{g}$ is the Lie algebra of $\mathcal{G}, \gamma \in J^{1} Y, \xi_{J^{1} Y}$ is the infinitesimal generator of the one parameter group of transformations of $J^{1} Y$ generated by $\xi_{Y} \in \mathfrak{g}$, and $\mathbf{i}(w)$ denotes the interior product by $w$. We denote $J^{\mathcal{L}}$ paired with $\xi_{Y}$ by $J^{\mathcal{L}}\left(\xi_{Y}\right)$ for short. This multimomentum map $J^{\mathcal{L}}$ is the key object that encodes the gauge transformation covariance of the theory.

If a section $\phi: X \rightarrow Y$ satisfies the Euler-Lagrange equations, then from (2.2) we obtain the (first) Noether theorem in the form:

$$
\mathbf{d}\left(\left(j^{1} \phi\right)^{*} J^{\mathcal{L}}\left(\xi_{Y}\right)\right)=0
$$

for all $\xi_{Y} \in \mathfrak{g}$.

Fix coordinates $x^{\mu}$ on $X,\left(x^{\mu}, y^{A}\right)$ on $Y$, and $\left(x^{\mu}, y^{A}, v^{A}{ }_{\mu}\right)$ on $J^{1} Y$, and write $\partial_{\mu}=\partial / \partial x^{\mu}, \partial_{A}=\partial / \partial y^{A}$, and $\partial_{A}{ }^{\mu}=\partial / \partial v^{A}{ }_{\mu}$. Set $d^{n+1} x=d x^{1} \wedge \ldots \wedge d x^{n+1}$ and $d^{n} x_{\mu}=\mathbf{i}\left(\partial_{\mu}\right) d^{n+1} x$, etc.. Then define $L$ by the local representation

$$
\mathcal{L}=L d^{n+1} x
$$

so that the Cartan form is given by

$$
\Theta_{\mathcal{L}}=L_{A}{ }^{\mu} d y^{A} \wedge d^{n} x_{\mu}+\left(L-L_{A}{ }^{\nu} v^{A}{ }_{\nu}\right) d^{n+1} x
$$

where $L_{A}^{\mu}:=\partial L / \partial v^{A}{ }_{\mu}$. For $\xi_{Y} \in \mathfrak{g}$, write

$$
\xi_{Y}=\xi^{\mu}\left(x^{\nu}\right) \partial_{\mu}+\xi^{A}\left(x^{\nu}, y^{B}\right) \partial_{A},
$$

\footnotetext{
${ }^{2}$ In most instances, the image of $\mathcal{G}$ is larger than $\operatorname{Diff}_{c}(X)$ (but still not necessarily all of Diff $(X)$ ). For our purposes, the precise nature of the image is unimportant, provided it contains $\operatorname{Diff}_{c}(X)$. We will elaborate these points later.

${ }^{3}$ Theories which are not parametrized can usually be made so by the simple expedients of, e.g., introducing a metric on $X$ and treating it as an auxiliary variable (either dynamic or not), or by coupling it to gravity.
} 
so that

$$
\xi_{J^{1} Y}=\xi^{\mu} \partial_{\mu}+\xi^{A} \partial_{A}+\left(D_{\mu} \xi^{A}-v^{A}{ }_{\nu} \xi^{\nu}, \mu\right) \partial_{A}{ }^{\mu}
$$

where $D_{\mu}=\partial_{\mu}+v_{\mu}^{A} \partial_{A}$ is the total derivative. Infinitesimally, (2.1) implies

$$
\left(L \xi^{\nu}\right)_{, \nu}+L_{A} \xi^{A}+L_{A}^{\mu}\left(D_{\mu} \xi^{A}-v^{A}{ }_{\nu} \xi_{, \mu}^{\nu}\right)=0
$$

for all $\xi_{Y} \in \mathfrak{g}$, where $L_{A}:=\partial L / \partial y^{A}$. Substituting the expression for $\xi_{J^{1} Y}$ and that for $\Theta_{\mathcal{L}}$ into (2.2) gives

$$
J^{\mathcal{L}}\left(\xi_{Y}\right)=\left(L_{A}{ }^{\mu} \xi^{A}+\left[L-L_{A}{ }^{\nu} v^{A}{ }_{\nu}\right] \xi^{\mu}\right) d^{n} x_{\mu}-L_{A}{ }^{\mu} \xi^{\nu} d y^{A} \wedge d^{n-1} x_{\mu \nu} .
$$

\section{The Stress-Energy-Momentum Tensor}

The SEM tensor measures the response of the fields to localized (i.e., compactly supported) spacetime deformations. However, $\operatorname{Diff}_{c}(X)$ does not necessarily act on the fields, at least not $a b$ initio. The reason is that $\operatorname{Diff}_{c}(X)$ is not naturally a subgroup of $\mathcal{G}$, but rather is a subgroup of the quotient group $\mathcal{G} / \mathcal{G}_{\text {Id }}$, where $\mathcal{G}_{\text {Id }}$ consists of those elements of $\mathcal{G}$ that cover the identity on $X$. Therefore, to define the SEM tensor, we need a group embedding $\operatorname{Diff}_{c}(X) \rightarrow \mathcal{G}$, so that each element of $\operatorname{Diff}_{c}(X)$ gives rise to a gauge transformation. To be precise, assume there is a $\mathcal{G}$-equivariant embedding of $\operatorname{Diff}_{c}(X)$ into $\mathcal{G}$ such that the composition $\operatorname{Diff}_{c}(X) \rightarrow \mathcal{G} \rightarrow \operatorname{Diff}(X)$ is the identity. (Note that the map $\operatorname{Diff}_{c}(X) \rightarrow \mathcal{G}$ is then a group homomorphism.) In general, $\mathcal{G}$ is larger than $\operatorname{Diff}_{c}(X)$, but the stress-energymomentum tensor is associated only with the $\operatorname{Diff}_{c}(X)$ "part" of $\mathcal{G}$. For instance, in continuum mechanics, $\mathcal{T}^{\mu \nu}$ is to measure the net energy flow, momentum flux and stress across hypersurfaces in spacetime - even if the material has internal structure.

\section{Remark}

1 Embeddings of $\operatorname{Diff}_{c}(X)$ into $\operatorname{Aut}(Y)$ exist if $Y$ is a "bundle of geometric objects," $c f$. Ferraris and Francaviglia [1983]. For example, if we have a tensor field theory, $\operatorname{Diff}_{c}(X)$ embeds into Aut $(Y)$ by push-forward: $\eta$ induces $\eta_{Y}=\eta_{*}=\left(\eta^{-1}\right)^{*}$. For Yang-Mills theories on the other hand, one may not be able to globally construct such embeddings. However, it will be clear from what follows that we really only require such embeddings to exist infinitesimally (i.e., on the Lie algebra level), and locally (i.e., over locally trivializing neighborhoods in $X$ ).

The embedding $\operatorname{Diff}_{c}(X) \rightarrow \mathcal{G}$ of Lie groups determines, by differentiation, a Lie algebra monomorphism $\mathfrak{X}_{c}(X) \rightarrow \mathfrak{g}$ given by $\xi \mapsto \xi_{Y}$, where in (2.4) $\xi^{A}=$ $\xi^{A}\left(x^{\mu}, y^{B},\left[\xi^{\nu}\right]\right)$ is a smooth function of $\xi^{\nu}$. We suppose that the action of $\operatorname{Diff}_{c}(X)$ on $Y$ is "local" in the sense that $\xi^{A}$ depends on $\xi^{\nu}$ and its derivatives up to order 
$k<\infty .{ }^{4}$ We call $k$ the differential index of the field theory; it is the order of the highest derivatives that appear in the transformation laws for the fields. The association $\xi \mapsto \xi_{Y}$ is linear in $\xi^{\nu}$, so we may write

$$
\xi^{A}=C_{\nu}^{A \rho_{1} \ldots \rho_{k}} \xi_{, \rho_{1} \ldots \rho_{k}}^{\nu}+\ldots+C_{\nu}^{A \rho} \xi_{, \rho}^{\nu}+C_{\nu}^{A} \xi^{\nu}
$$

where the coefficients $C_{\nu}^{A \rho_{1} \ldots \rho_{m}}$ depend only upon $x^{\mu}$ and $y^{B}$.

For tensor field theories, using the standard embedding $\eta \mapsto \eta_{*}$, one has simply

$$
\xi^{A}=C_{\nu}^{A \rho} \xi_{, \rho}^{\nu},
$$

so $k=1$ in this instance (unless $\phi$ is a scalar field, in which case all the coefficient functions $C_{\nu}^{A \rho_{1} \ldots \rho_{m}}$ vanish). But if one uses a "nonstandard" embedding $\operatorname{Diff}_{c}(X) \rightarrow$ $\mathcal{G}$, zeroth order terms may appear as well:

$$
\xi^{A}=C_{\nu}^{A \rho} \xi_{, \rho}^{\nu}+C_{\nu}^{A} \xi^{\nu} .
$$

We will illustrate this in the context of electromagnetism in $\S \mathbf{5}$. A Kirchhoff-Love rod under simple torsion provides a nontrivial example of a $k=0$ theory; cf. Love [1944]. For a field theory based on a linear connection on $X$ (like Palatini gravity), one would have $k=2$.

Before proceeding to the definition of the SEM tensor, we derive the consequences of the covariance condition (2.5). Substituting (3.1) into (2.5) yields

$$
\begin{gathered}
L_{, \nu} \xi^{\nu}+L \xi_{, \nu}^{\nu}+L_{A}\left(C_{\nu}^{A \rho_{1} \ldots \rho_{k}} \xi^{\nu}{ }_{, \rho_{1} \ldots \rho_{k}}+\ldots+C_{\nu}^{A \rho} \xi^{\nu}{ }_{, \rho}+C^{A}{ }_{\nu} \xi^{\nu}\right) \\
+L_{A}{ }^{\mu}\left(C_{\nu}^{A \rho_{1} \ldots \rho_{k}} \xi^{\nu}{ }_{, \rho_{1} \ldots \rho_{k} \mu}+\left(D_{\mu} C^{A \rho_{1} \ldots \rho_{k}}\right) \xi^{\nu}{ }_{, \rho_{1} \ldots \rho_{k}}\right. \\
\left.+\ldots+C^{A}{ }_{\nu} \xi^{\nu}{ }_{, \mu}+\left(D_{\mu} C^{A}{ }_{\nu}\right) \xi^{\nu}-v^{A}{ }_{\nu} \xi_{, \mu}^{\nu}\right)=0 .
\end{gathered}
$$

Since $\operatorname{Diff}_{c}(X) \rightarrow \mathcal{G}$ is an embedding, $\xi^{\nu}$ and its derivatives are arbitrarily specifiable. Thus, equating to zero the coefficients of the $\xi^{\nu}, \rho_{1} \ldots \rho_{m}$, we obtain the following results.

For $m=k+1$ :

$$
C_{\nu}^{A\left(\rho_{1} \ldots \rho_{k}\right.} L_{A}^{\left.\rho_{k+1}\right)}=0
$$

For $1<m<k+1$ :

$$
\left.C_{\nu}^{A \rho_{1} \ldots \rho_{m}} L_{A}+C_{\nu}^{A\left(\rho_{1} \ldots \rho_{m-1}\right.} L_{A} \rho_{m}\right)+\left(D_{\mu} C_{\nu}^{A \rho_{1} \ldots \rho_{m}}\right) L_{A}^{\mu}=0 .
$$

For $m=1$ :

$$
\left(L \delta^{\rho}{ }_{\nu}-L_{A} \rho_{v^{A}}^{A}\right)+C_{\nu}^{A \rho} L_{A}+C_{\nu}^{A} L_{A}{ }^{\rho}+\left(D_{\mu} C_{\nu}^{A \rho}\right) L_{A}{ }^{\mu}=0 .
$$

\footnotetext{
${ }^{4}$ The theory of Palais and Terng [1977] and Epstein and Thurston [1979] shows that locality in the sense of topology implies locality in the sense of pointwise dependence on a finite number of derivatives.
} 
For $m=0$ :

$$
L_{, \nu}+C^{A}{ }_{\nu} L_{A}+\left(D_{\mu} C^{A}{ }_{\nu}\right) L_{A}{ }^{\mu}=0 .
$$

Using the Leibniz rule, these can be rewritten

$$
\begin{gathered}
\left.C_{\nu}^{A\left(\rho_{1} \ldots \rho_{k}\right.} L_{A}{ }^{\rho_{k+1}}\right)=0 \\
C_{\nu}^{A\left(\rho_{1} \ldots \rho_{m-1}\right.} L_{A}{ }^{\left.\rho_{m}\right)}+D_{\mu}\left(C_{\nu}^{A \rho_{1} \ldots \rho_{m}} L_{A}{ }^{\mu}\right)+C_{\nu}^{A \rho_{1} \ldots \rho_{m}} \frac{\delta L}{\delta y^{A}}=0 \\
\left(L \delta^{\rho}{ }_{\nu}-L_{A}{ }^{\rho} v^{A}{ }_{\nu}\right)+C^{A}{ }_{\nu} L_{A}{ }^{\rho}+D_{\mu}\left(C_{\nu}^{A \rho} L_{A}{ }^{\mu}\right)+C_{\nu}^{A \rho} \frac{\delta L}{\delta y^{A}}=0 \\
L_{, \nu}+D_{\mu}\left(C^{A}{ }_{\nu} L_{A}{ }^{\mu}\right)+C^{A} \frac{\delta L}{\delta y^{A}}=0
\end{gathered}
$$

where $\delta L / \delta y^{A}=\partial L / \partial y^{A}-D_{\mu} L_{A}{ }^{\mu}$ is the variational derivative. Now set

$$
K_{\nu}^{\rho_{1} \ldots \rho_{m} \mu}:=C_{\nu}^{A \rho_{1} \ldots \rho_{m}} L_{A}^{\mu}
$$

note that $K_{\nu}^{\rho_{1} \ldots \rho_{m} \mu}$ is symmetric in all its contravariant indices except for the last. From (3.3) we obtain

$$
\left.\begin{array}{c}
K_{\nu}^{\left(\rho_{1} \ldots \rho_{k+1}\right)}=0 \\
K_{\nu}^{\left(\rho_{1} \ldots \rho_{m}\right)}+D_{\mu} K_{\nu}^{\rho_{1} \ldots \rho_{m} \mu} \approx 0 \\
\left(L \delta_{\nu}^{\rho}-L_{A}{ }^{\rho} v^{A}{ }_{\nu}\right)+K_{\nu}^{\rho}+D_{\mu} K_{\nu}^{\rho \mu} \approx 0 \\
L_{, \nu}+D_{\mu} K_{\nu}^{\mu} \approx 0
\end{array}\right\}
$$

where " $\approx$ " means "weak" equality, i.e., modulo the Euler-Lagrange equations.

We are now ready to state a main result, which characterizes fluxes of $J^{\mathcal{L}}$ across hypersurfaces in $X$.

Theorem 3.1. Consider a $\mathcal{G}$-covariant Lagrangian field theory. If $\phi$ is a solution of the Euler-Lagrange equations, then there exists a unique $(1,1)$-tensor density $\mathcal{T}(\phi)$ on $X$ such that

$$
\int_{\Sigma} i_{\Sigma}^{*}\left(j^{1} \phi\right)^{*} J^{\mathcal{L}}\left(\xi_{Y}\right)=\int_{\Sigma} \mathcal{T}^{\mu}{ }_{\nu}(\phi) \xi^{\nu} d^{n} x_{\mu}
$$

for all $\xi \in \mathfrak{X}_{c}(X)$ and all hypersurfaces $\Sigma$, where $i_{\Sigma}: \Sigma \rightarrow X$ is the inclusion.

Although this theorem presupposes that an embedding $\operatorname{Diff}_{c}(X) \rightarrow \mathcal{G}$ has been chosen, it will follow from Proposition $\mathbf{3}$ below that this choice is ultimately irrelevant. 
We call $\mathcal{T}^{\mu}{ }_{\nu}(\phi)$ the SEM tensor density of the field $\phi$. Before we proceed with the proof, we pause to demonstrate heuristically why $\mathcal{T}^{\mu}{ }_{\nu}$ so defined can be interpreted as a SEM tensor.

Consider a field $\psi$ coupled to gravity, so that the total Lagrangian density splits into matter and gravitational terms as follows:

$$
\mathcal{L}=\mathcal{L}_{m}+\mathcal{L}_{g},
$$

where $g$ is the spacetime metric and $\mathcal{L}_{g}=(1 / 16 \pi) R \sqrt{-g}$. (The precise nature of the coupling to matter is unimportant.) Suppose that the pair $(\psi, g)$ satisfies the Euler-Lagrange equations. From the Vanishing Theorem (see Remark 6 and equation (3.15) below) it follows that

$$
\int_{\Sigma} i_{\Sigma}^{*} j^{1}(\psi, g)^{*} J^{\mathcal{L}}\left(\xi_{Y}\right)=0
$$

for all $\xi \in \mathfrak{X}_{c}(X)$. From (2.6), $J^{\mathcal{L}}$ is linear in $\mathcal{L}$, so (3.6) can be rewritten

$$
-\int_{\Sigma} i_{\Sigma}^{*} j^{1}(\psi, g)^{*} J^{\mathcal{L}_{g}}\left(\xi_{Y}\right)=\int_{\Sigma} i_{\Sigma}^{*} j^{1}(\psi, g)^{*} J^{\mathcal{L}_{m}}\left(\xi_{Y}\right) .
$$

A straightforward calculation ${ }^{5}$ shows that

$$
-\int_{\Sigma} i_{\Sigma}^{*} j^{1}(\psi, g)^{*} J^{\mathcal{L}_{g}}\left(\xi_{Y}\right)=\frac{1}{8 \pi} \int_{\Sigma} \mathfrak{G}^{\mu}{ }_{\nu} \xi^{\nu} d^{n} x_{\mu}
$$

where $\mathfrak{G}^{\mu}{ }_{\nu}$ is the Einstein tensor density. From Einstein's equations $\mathfrak{G}^{\mu}{ }_{\nu}=8 \pi \mathfrak{T}^{\mu}{ }_{\nu}$ and (3.7), we obtain

$$
\int_{\Sigma} i_{\Sigma}^{*} j^{1}(\psi, g)^{*} J^{\mathcal{L}_{m}}\left(\xi_{Y}\right)=\int_{\Sigma} \mathfrak{T}^{\mu}{ }_{\nu} \xi^{\nu} d^{n} x_{\mu}
$$

so that in this case $\mathcal{T}^{\mu}{ }_{\nu}$ defined by (3.4) - with $\mathcal{L}_{m}$ in place of $\mathcal{L}$ - can be identified with the Hilbert SEM tensor density.

This actually constitutes a proof of Theorem $\mathbf{1}$ in the stated circumstances. ${ }^{6}$ But our setup here is more inclusive than fields coupled to gravity, and so we provide a proof in all generality.

Proof The pattern of the proof is the same for all $k$, so we shall be content with illustrating it for $k=2$. Using (2.6) and (3.1), the left hand side of (3.4) becomes

$$
\begin{aligned}
\int_{\Sigma} i_{\Sigma}^{*}\left(j^{1} \phi\right)^{*} J^{\mathcal{L}}\left(\xi_{Y}\right) & \\
= & \int_{\Sigma}\left(L_{A}{ }^{\mu}\left(\xi^{A}-v^{A}{ }_{\nu} \xi^{\nu}\right)+L \xi^{\mu}\right) d^{n} x_{\mu} \\
= & \int_{\Sigma}\left(L_{A}{ }^{\mu}\left(C_{\nu}^{A \rho \tau} \xi^{\nu}{ }_{, \rho \tau}+C_{\nu}^{A \rho} \xi_{, \rho}^{\nu}+C^{A}{ }_{\nu} \xi^{\nu}-v^{A}{ }_{\nu} \xi^{\nu}\right)+L \delta^{\mu}{ }_{\nu} \xi^{\nu}\right) d^{n} x_{\mu} \\
= & \int_{\Sigma}\left(K_{\nu}^{\rho \tau \mu} \xi_{, \rho \tau}^{\nu}+K_{\nu}^{\rho \mu} \xi_{, \rho}^{\nu}+\left(L \delta^{\mu}{ }_{\nu}-L_{A}{ }^{\mu} v^{A}{ }_{\nu}+K_{\nu}^{\mu}\right) \xi^{\nu}\right) d^{n} x_{\mu} .
\end{aligned}
$$

\footnotetext{
${ }^{5}$ See Gotay et al. [1992] for details.

${ }^{6}$ Another proof will be given in $\S \mathbf{4}$.
} 
Let $U \subset X$ be a chart in which $\Sigma \cap U$ is a hyperplane. By means of a partition of unity argument, it suffices to consider the case when the vector field $\xi$ has support contained in $U$. Construct an $(n+1)$-dimensional region $V \subset X$ such that $\partial V=$ $(\Sigma \cap U) \cup \Sigma^{\prime}$, where $\xi \mid \Sigma^{\prime}=0$. By the divergence theorem, the first two terms in (3.9) become

$$
\begin{aligned}
\int_{\Sigma} & \left(K_{\nu}^{\rho \tau \mu} \xi_{, \rho \tau}^{\nu}+K_{\nu}^{\rho \mu} \xi_{, \rho}^{\nu}\right) d^{n} x_{\mu} \\
= & \int_{V} D_{\mu}\left(K_{\nu}^{\rho \tau \mu} \xi_{, \rho \tau}^{\nu}+K_{\nu}^{\rho \mu} \xi_{, \rho}^{\nu}\right) d^{n+1} x \\
= & \int_{V}\left[\left(D_{\mu} K_{\nu}^{\rho \tau \mu}\right) \xi_{, \rho \tau}^{\nu}+K_{\nu}^{\rho \tau \mu} \xi_{, \rho}^{\nu}{ }_{, \rho}\right. \\
& \left.\quad+\left(D_{\mu} K_{\nu}^{\rho \mu}\right) \xi_{, \rho}^{\nu}+K_{\nu}^{\rho \mu} \xi^{\nu}{ }_{, \rho \mu}\right] d^{n+1} x .
\end{aligned}
$$

In (3.9) the second term vanishes by virtue of the first equation in (3.4) and, using (3.4b), the first and fourth terms cancel. Applying the Leibniz rule to the third term yields

$$
\int_{V}\left(D_{\mu} K_{\nu}^{\rho \mu}\right) \xi_{, \rho}^{\nu} d^{n+1} x=\int_{V}\left\{D_{\rho}\left[\left(D_{\mu} K_{\nu}^{\rho \mu}\right) \xi^{\nu}\right]+\left(D_{\rho} D_{\mu} K_{\nu}^{\rho \mu}\right) \xi^{\nu}\right\} d^{n+1} x .
$$

Using (3.4b) once more, the second integrand here can be rewritten

$$
\left(D_{\rho} D_{\mu} K_{\nu}^{\rho \mu}\right) \xi^{\nu}=-\left(D_{\rho} D_{\mu} D_{\tau} K_{\nu}^{\rho \mu \tau}\right) \xi^{\nu}
$$

which vanishes by symmetry, $c f$. (3.4a). Thus (3.9) reduces to

$$
\int_{V} D_{\rho}\left[\left(D_{\mu} K_{\nu}^{\rho \mu}\right) \xi^{\nu}\right] d^{n+1} x=\int_{\Sigma}\left(D_{\mu} K_{\nu}^{\rho \mu}\right) \xi^{\nu} d^{n} x_{\rho}
$$

again by the divergence theorem.

Substituting these results back into (3.9) and reindexing, we therefore obtain (3.5) with

$$
\mathcal{T}^{\mu}{ }_{\nu}=L \delta^{\mu}{ }_{\nu}-L_{A}{ }^{\mu} v^{A}{ }_{\nu}+K_{\nu}^{\mu}+D_{\rho} K_{\nu}^{\mu \rho}
$$

Reverting to our original notation, this last formula becomes

$$
\mathcal{T}^{\mu}{ }_{\nu}=L \delta^{\mu}{ }_{\nu}-L_{A}{ }^{\mu} v^{A}{ }_{\nu}+L_{A}{ }^{\mu} C^{A}{ }_{\nu}+D_{\rho}\left(L_{A}{ }^{\rho} C_{\nu}^{A \mu}\right) .
$$

Taking (1.1) into account, we see that

$$
\mathcal{T}^{\mu}{ }_{\nu}=\mathfrak{t}^{\mu}{ }_{\nu}+\text { "correction terms"; }
$$

hence $\mathcal{T}^{\mu}{ }_{\nu}$ may be regarded as a modification of the canonical SEM tensor density. Formula (3.11) is thus a generalized Belinfante-Rosenfeld formula. Although we have derived (3.11) in the case when $k=2$, this expression is valid for any $k$. 
We emphasize that (3.4) uniquely determines $\mathcal{T}^{\mu}{ }_{\nu}$. This is a decided virtue of $(i)$ defining $\mathcal{T}^{\mu}{ }_{\nu}$ by means of fluxes, and (ii) using the full spacetime diffeomorphism group in our constructions as opposed to just translations.

\section{Remarks}

2 Formula (3.11) is consistent with (1.1) if one uses, instead of $\operatorname{Diff}_{c}(X)$, just translations in $X$ and sets $\xi^{A}=0$. Similarly, if one uses the Poincaré group in place of $\operatorname{Diff}_{c}(X)$ then, for tensor field theories, $\mathcal{T}^{\mu}{ }_{\nu}$ reduces to the canonical SEM tensor as modified by Belinfante [1939]; see also Wentzel [1949] and Corson [1953]. However, with our approach the "correction terms" in (3.11) naturally appear. We mention that Taub [1970] makes use of a special version of formula (3.12) (see his equation (5.3)) in his studies of variational principles and stability.

3 It may seem odd that only terms corresponding to the zeroth and first order derivatives in (3.1) appear in (3.12). The contributions from higher order derivatives are actually hidden in the symmetric part $D_{\rho}\left(C_{\nu}^{A(\mu} L_{A}^{\rho)}\right)$ of $D_{\rho}\left(C_{\nu}^{A \mu} L_{A} \rho\right)$. Indeed, when $k=1$, it follows from (3.3a) that this latter quantity is antisymmetric in $\mu$ and $\rho$; thus, the antisymmetric part $D_{\rho}\left(C_{\nu}^{A[\mu} L_{A}{ }^{\rho]}\right)$ represents the "pure" first derivative corrections. Now suppose that $k=2$. Applying (3.3b) and (3.3a), one sees that

$$
D_{\rho}\left(C_{\nu}^{A(\mu} L_{A}^{\rho)}\right)=-D_{\rho} D_{\tau}\left(C_{\nu}^{A \mu \rho} L_{A}^{\tau}\right)=\frac{1}{2} D_{\rho} D_{\tau}\left(C_{\nu}^{A \rho \tau} L_{A}{ }^{\mu}\right) .
$$

Thus $\mathcal{T}^{\mu}{ }_{\nu}$ can be expressed in terms of all the higher derivative coefficients as follows:

$$
\mathcal{T}^{\mu}{ }_{\nu}=L \delta^{\mu}{ }_{\nu}-L_{A}{ }^{\mu} v^{A}{ }_{\nu}+L_{A}{ }^{\mu} C^{A}{ }_{\nu}-D_{\rho}\left(C_{\nu}^{A[\rho} L_{A}{ }^{\mu]}\right)+\frac{1}{2} D_{\rho} D_{\tau}\left(C_{\nu}^{A \rho \tau} L_{A}{ }^{\mu}\right) .
$$

This formula is equivalent to (3.12) when $k=2$; for higher indices $k$ one obtains analogous formulae by iteratively applying $(3.3 \mathrm{a}, \mathrm{b})$, all of which can be succinctly subsumed under (3.12).

4 Note that, of the four covariance conditions (3.4a-d), we have so far used only the first two. This observation implies that the main hypothesis in Theorem $\mathbf{1}$, viz. that the field $\phi$ satisfy the Euler-Lagrange equations, can often be eliminated. Indeed, in the proof of Theorem 1 the Euler-Lagrange equations come into play only through applications of the covariance conditions (3.4b). But these conditions are vacuous for field theories with $k \leq 1$. This is an important point, since most field theories - in particular tensor and spinor field theories - have index 1, and hence in such cases no restriction need be placed on the section $\phi$ in order to construct the SEM tensor.

5 As noted in $\S \mathbf{2}$, the image of $\mathcal{G}$ under the natural projection $\operatorname{Aut}(Y) \rightarrow \operatorname{Diff}(X)$ may be strictly larger than $\operatorname{Diff}_{c}(X)$. Let us denote this image by $\mathcal{D}\left(=\mathcal{G} / \mathcal{G}_{\text {Id }}\right)$. In such cases one could consider an embedding $\mathcal{D} \rightarrow \mathcal{G}$. But then the relation (3.5) between the integals of $J^{\mathcal{L}}$ and $\mathcal{T}^{\mu}{ }_{\nu}$ for general vector fields $\xi$ in the Lie algebra 
of $\mathcal{D}$ might only hold modulo surface terms. For instance, suppose that $k=1$ and that $\mathcal{D}=\operatorname{Diff}(X)$. Tracing back through the proof of Theorem 1, and using Stokes' theorem in place of the divergence theorem, we obtain

$$
\int_{\Sigma} i_{\Sigma}^{*}\left(j^{1} \phi\right)^{*} J^{\mathcal{L}}\left(\xi_{Y}\right)=\int_{\Sigma} \mathcal{T}^{\mu}{ }_{\nu} \xi^{\nu} d^{n} x_{\mu}+\frac{1}{2} \int_{\partial \Sigma} L_{A}{ }^{\mu} C_{\nu}^{A \rho} \xi^{\nu} d^{n-1} x_{\mu \rho}
$$

for $\xi \in \mathfrak{X}(X)$. (In this computation we have used the fact that, for $k=1$, the quantity $L_{A}{ }^{\mu} C_{\nu}^{A \rho}$ is antisymmetric in $\mu$ and $\rho$, cf. Remark 3.) In the asymptotically flat context, it is plausible that surface terms such as the one in this expression could be identified with the energy and momentum of the gravitational field. (See the gravity example in $\S \mathbf{5}$ for further discussion of this point.)

In any event we emphasize that Theorem $\mathbf{1}$ as stated is valid regardless of what $\mathcal{D}$ is, as long as $\operatorname{Diff}_{c}(X) \subset \mathcal{D}$, and thus Theorem $\mathbf{1}$ can always be used to define $\mathcal{T}^{\mu}{ }_{\nu}$.

Before proceeding with a discussion of $\mathcal{T}^{\mu}{ }_{\nu}$ and its properties, we want to broaden our formalism to encompass certain situations that commonly arise in applications. Suppose that the fields consist of two types: $\phi^{A}=\left(\psi^{a}, \chi^{s}\right)$, where the $\psi^{a}$ are dynamic and the $\chi^{s}$ are not. We have in mind theories on a nondynamic spacetime, in which context the $\chi^{s}$ might comprise a metric or a tetrad, or we are dealing with matter fields in the presence of a nondynamic Yang-Mills field, etc.. In such cases the $\chi^{s}$ appear as parameters in the Lagrangian density, in the sense that they can take on different values ${ }^{7}$ but are not varied to give field equations.

In practice, one finds that the nondynamic fields have certain special characteristics, and enter the theory in a relatively uncomplicated manner. Reflecting these empirical facts, we make the following Ansätze:

(A1) The $\chi^{s}$ have index $k \leq 1$, and

(A2) the $\chi^{s}$ do not derivatively couple in the Lagrangian density.

The second Ansatz means that $\mathcal{L}$ depends only upon the first jet of the $\psi^{a}$ and on point values of the $\chi^{s}$. Although (A2) can be lifted in various circumstances (e.g., when $\chi^{s}$ is a metric on spacetime, $\left.c f . \S 4\right)$, it is generally believed that derivatively coupled theories are, to a large extent, pathological.

Upon separating the dynamic from the nondynamic fields we may, as before, peel off various consequences of the infinitesimal equivariance condition (2.5). But now we must be careful, as the $\chi^{s}$ do not satisfy any Euler-Lagrange equations. Since by (A1) the $\chi^{s}$ have index $k \leq 1$, this circumstance does not affect $(3.4 \mathrm{a}, \mathrm{b})$, which remain valid as stated. It follows that Theorem $\mathbf{1}$ also remains valid in this context, as its derivation depends only upon (3.4a,b), cf. Remark 4. In particular, $\mathcal{T}^{\mu}{ }_{\nu}$ is still given by (3.12). However, (3.4c) - corresponding to the coefficients of the first derivatives of $\xi^{\nu}$ in (3.1) - must be modified. From (3.3c) and (3.12) we obtain the generalized Hilbert formula

$$
\mathcal{T}^{\mu}{ }_{\nu}=-C_{\nu}^{s \mu} \frac{\delta L}{\delta y^{s}}
$$

\footnotetext{
${ }^{7}$ As they usually must, in order to keep the theory $\mathcal{G}$-covariant; this is in keeping with our requirement that the system be parametrized.
} 
When $\chi^{s}$ is actually a metric on spacetime we will show in $\S 4$ that (3.12) reduces to $(1.2)$.

Since the $\chi^{s}$ are nondynamic, the first Noether theorem (2.3) may not hold. In terms of $\mathcal{T}^{\mu}{ }_{\nu}$, this is evidenced by the following result.

\section{Proposition 3.2.}

$$
D_{\mu} \mathcal{T}^{\mu}{ }_{\nu}=\left(v^{s}{ }_{\nu}-C^{s}{ }_{\nu}\right) \frac{\delta L}{\delta y^{s}} .
$$

Proof There are various ways of seeing this. Here we give a proof that, although perhaps inelegant, highlights the role of the last covariance condition (3.3d), which we have not thus far used.

From (3.10),

$$
\begin{aligned}
D_{\mu} \mathcal{T}_{\nu}^{\mu}= & D_{\mu}\left(L \delta^{\mu}{ }_{\nu}-L_{A}{ }^{\mu} v^{A}{ }_{\nu}+K_{\nu}^{\mu}+D_{\rho} K_{\nu}^{\mu \rho}\right) \\
= & \left(L_{, \nu}+L_{A} v^{A}{ }_{\nu}+L_{A}{ }^{\mu} v^{A}{ }_{\mu \nu}-\left(D_{\mu} L_{A}{ }^{\mu}\right) v^{A}{ }_{\nu}-L_{A}{ }^{\mu} v^{A}{ }_{\nu \mu}\right. \\
& \left.+D_{\mu} K_{\nu}^{\mu}+D_{\mu} D_{\rho} K_{\nu}^{\mu \rho}\right)
\end{aligned}
$$

where $v^{A}{ }_{\mu \nu}\left(j^{1} \phi\right)=\phi^{A}{ }_{, \mu \nu}$. Here the third and fifth terms cancel while the second and fourth combine to produce a variational derivative. Using (3.4b,a) repeatedly, the last term is seen to vanish. Thus we obtain

$$
D_{\mu} \mathcal{T}_{\nu}^{\mu}=\left(L_{, \nu}+D_{\mu} K_{\nu}^{\mu}+\frac{\delta L}{\delta y^{A}} v^{A}{ }_{\nu}\right) .
$$

The last term can be expanded

$$
\frac{\delta L}{\delta y^{A}} v^{A}{ }_{\nu}=\frac{\delta L}{\delta y^{a}} v^{a}{ }_{\nu}+\frac{\delta L}{\delta y^{s}} v_{\nu}^{s} .
$$

The first of these vanishes by the Euler-Lagrange equations. Finally, according to (3.3d), the first two terms in (3.14) sum to

$$
-C^{s}{ }_{\nu} \frac{\delta L}{\delta y^{s}}
$$

Combining these computations yields (3.13).

One of the main advantages of our construction is that $\mathcal{T}(\phi)$ is both gauge and embedding independent. To prove these results we need the following lemma. Let $\mathfrak{g}_{\text {Id }}$ be the Lie algebra of $\mathcal{G}_{\text {Id }}$; it consists of all gauge generators on $Y$ which are $\pi_{X Y}$-vertical.

Lemma 3.3. Consider a $\mathcal{G}$-covariant field theory. Let $\zeta_{Y} \in \mathfrak{g}_{\mathrm{Id}}$ be compactly supported along $X$. Then

$$
\int_{\Sigma} i_{\Sigma}^{*}\left(j^{1} \phi\right)^{*} J^{\mathcal{L}}\left(\zeta_{Y}\right)=0
$$

for all hypersurfaces $\Sigma$. 
Proof Without loss of generality we may suppose that $\zeta_{X}$ is supported in a chart in which $\Sigma$ is a hyperplane, so that we may apply the divergence theorem, as in the proof of Theorem 1. Then from (2.6) and the fact that $\zeta^{\mu}=0$, we have

$$
\begin{aligned}
\int_{\Sigma} i_{\Sigma}^{*}\left(j^{1} \phi\right)^{*} J^{\mathcal{L}}\left(\zeta_{Y}\right) & =\int_{V} \mathbf{d}\left(\left(j^{1} \phi\right)^{*} J^{\mathcal{L}}\left(\zeta_{Y}\right)\right) \\
& =\int_{V} D_{\mu}\left(L_{A}^{\mu} \zeta^{A}\right) d^{n+1} x \\
& =\int_{V}\left[\left(D_{\mu} L_{A}^{\mu}\right) \zeta^{A}+L_{A}^{\mu}\left(D_{\mu} \zeta^{A}\right)\right] d^{n+1} x
\end{aligned}
$$

Upon separating the dynamic from the nondynamic fields and taking Ansatz (A2) into account, the last integrand reduces to

$$
\left(D_{\mu}{L_{a}}^{\mu}\right) \zeta^{a}+L_{a}{ }^{\mu}\left(D_{\mu} \zeta^{a}\right)=\left(D_{\mu} L_{a}{ }^{\mu}-L_{a}\right) \zeta^{a}
$$

by (2.5). But this vanishes as the $\psi^{a}$ are dynamic.

Note that the lemma holds in complete generality, and in particular does not involve the embedding $\operatorname{Diff}_{c}(X) \rightarrow \mathcal{G}$.

\section{Remarks}

6 When all the fields are dynamic, it follows from the first Noether theorem (2.3) that

$$
\int_{\Sigma} i_{\Sigma}^{*}\left(j^{1} \phi\right)^{*} J^{\mathcal{L}}\left(\zeta_{Y}\right)=0
$$

for all $\zeta_{Y} \in \mathfrak{g}$ which are compactly supported along $X$, vertical or not. This result is known as the Vanishing Theorem or the (second) Noether theorem.

7 This lemma is the only occasion upon which we need to use Ansatz (A2) for the nondynamic fields.

Proposition 3.4. $\mathcal{T}(\phi)$ is gauge-covariant.

By this we mean that the tensor density $\mathcal{T}(\phi)$ satisfies

$$
\mathcal{T}\left(\eta_{Y} \cdot \phi\right)=\left(\eta_{X}\right)_{*} \mathcal{I}(\phi)
$$

for all $\eta_{Y} \in \mathcal{G}$. Here, the induced action of $\eta_{Y}$ on fields is $\eta_{Y} \cdot \phi=\eta_{Y} \circ \phi \circ \eta_{X}^{-1}$.

Proof First note that $j^{1}\left(\eta_{Y} \cdot \phi\right)=\eta_{J^{1}(Y)} \circ j^{1} \phi \circ \eta_{X}^{-1}$. Moreover, one readily verifies that $J^{\mathcal{L}}$ given by $(2.2)$ is $\mathcal{G}$-equivariant, so that

$$
\left(\eta_{J^{1} Y}\right)^{*} J^{\mathcal{L}}\left(\xi_{Y}\right)=J^{\mathcal{L}}\left(T \eta_{Y}^{-1} \cdot \xi_{Y}\right)
$$


for each $\xi_{Y} \in \mathfrak{g}$. Thus for all $\eta_{Y} \in \mathcal{G}$ and $\xi \in \mathfrak{X}_{c}(X)$, we have

$$
\begin{aligned}
\int_{\Sigma} \mathcal{T}^{\mu}{ }_{\nu}\left(\eta_{Y} \cdot \phi\right) \xi^{\nu} d^{n} x_{\mu} & =\int_{\Sigma} i_{\Sigma}^{*}\left(j^{1}\left(\eta_{Y} \cdot \phi\right)\right)^{*} J^{\mathcal{L}}\left(\xi_{Y}\right) \\
& =\int_{\Sigma} i_{\Sigma}^{*}\left(\eta_{X}^{-1}\right)^{*}\left(j^{1} \phi\right)^{*}\left(\eta_{J^{1} Y}\right)^{*} J^{\mathcal{L}}\left(\xi_{Y}\right) \\
& =\int_{\Sigma} i_{\Sigma}^{*}\left(\eta_{X}^{-1}\right)^{*}\left(j^{1} \phi\right)^{*} J^{\mathcal{L}}\left(T \eta_{Y}^{-1} \cdot \xi_{Y}\right) \\
& =\int_{\Sigma^{\prime}} i_{\Sigma^{\prime}}^{*}\left(j^{1} \phi\right)^{*} J^{\mathcal{L}}\left(T \eta_{Y}^{-1} \cdot \xi_{Y}\right)
\end{aligned}
$$

where $\Sigma^{\prime}=\eta_{X}^{-1}(\Sigma)$.

Now consider the difference $T \eta_{Y}^{-1} \cdot \xi_{Y}-\left(T \eta_{X}^{-1} \cdot \xi\right)_{Y}$. This vector field is $\pi_{X Y^{-}}$ vertical and compactly supported along $X$, and so the lemma implies

$$
\int_{\Sigma^{\prime}} i_{\Sigma^{\prime}}^{*}\left(j^{1} \phi\right)^{*} J^{\mathcal{L}}\left(T \eta_{Y}^{-1} \cdot \xi_{Y}\right)=\int_{\Sigma^{\prime}} i_{\Sigma^{\prime}}^{*}\left(j^{1} \phi\right)^{*} J^{\mathcal{L}}\left(\left(T \eta_{X}^{-1} \cdot \xi\right)_{Y}\right)
$$

Consequently

$$
\begin{aligned}
& \int_{\Sigma} \cdot \mathcal{T}^{\mu}{ }_{\nu}\left(\eta_{Y} \cdot \phi\right) \xi^{\nu} d^{n} x_{\mu}=\int_{\Sigma^{\prime}} i_{\Sigma^{\prime}}^{*}\left(j^{1} \phi\right)^{*} J^{\mathcal{L}}\left(\left(T \eta_{X}^{-1} \cdot \xi\right)_{Y}\right) \\
& =\int_{\Sigma^{\prime}} \mathcal{T}_{\nu^{\prime}}^{\mu^{\prime}}(\phi)\left(T \eta_{X}^{-1} \cdot \xi\right)^{\nu^{\prime}} d^{n} x_{\mu^{\prime}} \\
& =\int_{\Sigma} \mathcal{T}_{\nu^{\prime}}^{\mu^{\prime}}(\phi)\left(T \eta_{X}\right)_{\mu^{\prime}}^{\mu}\left(T \eta_{X}^{-1}\right)_{\nu}^{\nu^{\prime}} \operatorname{det}\left(T \eta_{X}^{-1}\right) \xi^{\nu} d^{n} x_{\mu}
\end{aligned}
$$

by the change of variables formula, and this yields (3.16).

When the gauge transformation $\eta_{Y}$ is purely "internal", i.e., $\eta_{Y} \in \mathcal{G}_{\mathrm{Id}}$, (3.16) reduces to $\mathcal{T}\left(\eta_{Y} \cdot \phi\right)=\mathcal{T}(\phi)$. Thus $\mathcal{T}(\phi)$ is actually gauge-invariant in this case. At the opposite extreme, when $\eta_{Y}$ is the lift of a diffeomorphism of $X,(3.16)$ reiterates the fact that $\mathcal{T}(\phi)$ is a tensor density.

A similar argument establishes:

Proposition 3.5. $\mathcal{T}^{\mu}{ }_{\nu}$ is independent of the choice of embedding $\operatorname{Diff}_{c}(X) \rightarrow \mathcal{G}$.

Proof Fix $\xi \in \mathfrak{X}_{c}(X)$ and let $\xi_{Y}$ and $\xi_{Y}^{\prime}$ be the images of $\xi$ in $\mathfrak{g}$ under the differentials of two such embeddings. Since the vertical difference $\xi_{Y}^{\prime}-\xi_{Y}$ is compactly supported along $X$, we may apply the lemma once again, thereby obtaining

$$
\int_{\Sigma} i_{\Sigma}^{*}\left(j^{1} \phi\right)^{*} J^{\mathcal{L}}\left(\xi_{Y}^{\prime}\right)=\int_{\Sigma} i_{\Sigma}^{*}\left(j^{1} \phi\right)^{*} J^{\mathcal{L}}\left(\xi_{Y}\right)
$$

The desired result now follows from (3.5).

Thus even though the last two terms in (3.12) individually depend upon the choice of embedding $\operatorname{Diff}_{c}(X) \rightarrow \mathcal{G}$, their sum does not. 
One can also inquire as to the dependence of $\mathcal{T}^{\mu}{ }_{\nu}$ upon the choice of Lagrangian density $\mathcal{L}$. As is well known, $\mathcal{L}$ is not uniquely fixed; one is free to add a $\mathcal{G}$-equivariant divergence to it without changing the physical content of the theory. We now prove that $\mathcal{T}^{\mu}{ }_{\nu}$, unlike $\mathfrak{t}^{\mu}{ }_{\nu}$, is independent of such ambiguities.

Proposition 3.6. $\mathcal{T}^{\mu}{ }_{\nu}$ depends only upon the divergence equivalence class of $\mathcal{L}$.

Proof Recall that a Lagrangian density $\mathcal{L}$ is a "pure divergence" if $\mathcal{L}=D \mathcal{M}$, where $\mathcal{M}$ is a bundle map $Y \rightarrow \Lambda^{n} X$ and $D$ is the horizontal exterior differential. (See Anderson [1992] for the relevant background.) Since $\mathcal{T}^{\mu}{ }_{\nu}$ depends linearly on $\mathcal{L}$, to prove Proposition 4 it suffices to show that the SEM tensor of a pure divergence Lagrangian density vanishes identically. But this follows from (3.5), the Vanishing Theorem (3.15), and the fact that every section $\phi: X \rightarrow Y$ is an extremal of a pure divergence Lagrangian.

If $\mathcal{T}^{\mu}{ }_{\nu}$ is to be the SEM tensor of a classical field theory, then one ought to be able to recover the Hamiltonian as the integral of $-\mathcal{T}^{0}{ }_{0}$ over a (compact and boundaryless) Cauchy surface. This works as follows. Let $\Sigma \subset X$ be such a Cauchy surface and fix a vector field $\zeta$ on (a neighborhood of $\Sigma$ in) $X$ with the property that $\zeta$ is transversal to $\Sigma$ and consider $\zeta_{Y}$; it can be thought of as defining an "evolution direction" on $Y$. From general principles (Gotay et al. [1992]), the Hamiltonian corresponding to the choice of evolution direction $\zeta_{Y}$ is given by

$$
\begin{aligned}
H_{\zeta} & =-\int_{\Sigma} i_{\Sigma}^{*}\left(j^{1} \phi\right)^{*} J^{\mathcal{L}}\left(\zeta_{Y}\right) \\
& =-\int_{\Sigma} \mathcal{T}^{\mu}{ }_{\nu} \zeta^{\nu} d^{n} x_{\mu} .
\end{aligned}
$$

Upon choosing adapted coordinates in which $\Sigma$ is locally the level set $x^{0}=0$ and $\zeta_{Y}=\partial / \partial x^{0}$, this reduces to the expected result. This result can also be obtained directly from (3.12) in adapted coordinates, although it must be noted that $-\mathcal{T}^{0}{ }_{0}$ in general cannot be identified with the Hamiltonian density, which of course is just $-\mathfrak{t}^{0}{ }_{0}$.

A notable feature of our SEM tensor $\mathcal{T}^{\mu}{ }_{\nu}(\phi)$ is that it vanishes on shell, i.e., when $\phi$ satisfies the Euler-Lagrange equations. This is a consequence of the third covariance condition (3.3c) and the expression (3.12) for $\mathcal{T}^{\mu}{ }_{\nu}(\phi)$. [Equivalently, this follows from (3.15) and (3.4).] While at first glance it may seem strange, a moment's reflection shows that the vanishing of $\mathcal{T}^{\mu}{ }_{\nu}(\phi)$ is a typical feature of parametrized theories in which all fields are dynamic. Indeed, one knows, for instance, that the Hamiltonian of such a theory is also zero on shell. ${ }^{8}$ To place this phenomenon in a more concrete setting, consider again the case of a matter field coupled to gravity. Then indeed the total Hamiltonian of the system vanishes on shell, although the matter Hamiltonian does not. Similarly, in the context of SEM tensors, one sees

\footnotetext{
${ }^{8}$ Provided $X$ is spatially closed. This can be seen directly from (3.18), but is true independently of any relation between the SEM tensor and the Hamiltonian.
} 
from the discussion immediately after the statement of Theorem 1 that the total SEM tensor $\mathcal{T}^{\mu}{ }_{\nu}$ constructed using the total Lagrangian density $\mathcal{L}$ is given by

$$
\mathcal{T}^{\mu}{ }_{\nu}=-\frac{1}{8 \pi} \mathfrak{G}^{\mu}{ }_{\nu}+\mathfrak{T}^{\mu}{ }_{\nu}
$$

which vanishes by virtue of Einstein's equations. Thus to surmount this "difficulty", one should construct the SEM tensor using not the total Lagrangian, but rather only its matter part $\mathcal{L}_{m}$, in which case one obtains $\mathcal{T}^{\mu}{ }_{\nu}=\mathfrak{T}^{\mu}{ }_{\nu}$ as expected. Decomposing the Lagrangian in the form (3.6) may or may not be straightforward, as illustrated in the examples in $\S \mathbf{5}$; in any event, we now turn to a detailed discussion of what happens when a metric is present on $X$.

\section{Coupling to a Metric}

Let $g$ be a metric on $X$, which may or may not be dynamic and, in any case, need not represent gravity. ${ }^{9}$ We do not make any assumptions on the nature of the coupling between the metric and the other fields, other than that the Lagrangian remain first order. Thus, in particular, the metric may couple derivatively ${ }^{10}$ and non-minimally to these other fields $\psi^{a}$, which we shall refer to collectively as "matter" fields, even though this appellation may be somewhat inappropriate in particular examples. In this section, $\mathcal{L}$ will always refer to the matter Lagrangian; a "free field" Lagrangian for the metric is immaterial. We assume that the $\psi^{a}$ are on shell.

Let $\operatorname{Diff}_{c}(X)$ act on $g$ by pushforward. Thus for $\xi \in \mathfrak{X}_{c}(X)$ the "metric component" of $\xi_{Y}$ is

$$
\left(\xi_{Y}\right)_{\alpha \beta}=-\left(g_{\nu \beta} \xi_{, \alpha}^{\nu}+g_{\nu \alpha} \xi_{, \beta}^{\nu}\right)
$$

hence

$$
C_{\alpha \beta \nu}{ }^{\rho}=-\left(g_{\nu \beta} \delta_{\alpha}^{\rho}+g_{\nu \alpha} \delta_{\beta}^{\rho}\right)
$$

is the only nonzero coefficient in (3.1). (Thus the metric is a pure index 1 field.)

Formula (3.12) gives

$$
\begin{aligned}
\mathcal{T}^{\mu}{ }_{\nu}= & L \delta^{\mu}{ }_{\nu}-L_{a}{ }^{\mu} v^{a}{ }_{\nu}+L_{a}{ }^{\mu} C^{a}{ }_{\nu}+D_{\rho}\left(L_{a}{ }^{\rho} C^{a \mu}{ }_{\nu}\right) \\
& -\frac{\partial L}{\partial g_{\alpha \beta \mu}} g_{\alpha \beta \nu}-2 D_{\rho}\left(\frac{\partial L}{\partial g_{\mu \beta \rho}} g_{\nu \beta}\right)
\end{aligned}
$$

where $g_{\alpha \beta \nu}\left("=g_{\alpha \beta, \nu} "\right)$ are the velocity variables associated to the $g_{\alpha \beta}$. On the other hand, from (3.13) and (4.1) we have

$$
\mathcal{T}^{\mu}{ }_{\nu}=-C_{\alpha \beta \nu}{ }^{\mu} \frac{\delta L}{\delta g_{\alpha \beta}}=2 \frac{\delta L}{\delta g_{\mu \rho}} g_{\nu \rho}
$$

\footnotetext{
${ }^{9}$ For instance, the bosonic string has a metric on $X$ which is dynamic, but which has naught to do with gravity. See $\S 5$.

${ }^{10}$ Despite Ansatz (A2), we may allow derivative couplings when the nondynamic field is a metric on $X$. This is because the metric transforms only by (some subgroup of) $\operatorname{Diff}(X)$ - which is "horizontal" in $\mathcal{G}$ - so that there is no metric contribution to $\mathcal{G}_{\text {Id }}$. Consequently Lemma $\mathbf{1}$ is trivially satisfied in this circumstance. We remark that the analogous assertion is not true for frames, which do have internal gauge freedom (viz. frame rotations).
} 
Raising indices then yields

\section{Theorem 4.1.}

$$
\mathcal{T}^{\mu \nu}=2 \frac{\delta L}{\delta g_{\mu \nu}}
$$

As a consequence, $\mathcal{T}^{\mu \nu}$ is manifestly symmetric, gauge-covariant, and independent of the choice of embedding - attributes that are hardly obvious from (4.2)! Furthermore $\mathcal{T}^{\mu \nu}$ need not vanish unless, of course, $g$ is dynamic as well as $\psi$. Note finally that (4.3) holds regardless of whether $g$ represents the gravitational field.

We derive one last consequence of our formalism.

Proposition 4.2. $\mathcal{T}^{\mu}{ }_{\nu}$ is covariantly conserved.

Proof Fix a point $x \in X$ and work in normal coordinates centered there. Since $g$ has pure index $1,(3.13)$ gives

$$
\nabla_{\mu} \mathcal{T}_{\nu}^{\mu}(x)=D_{\mu} T_{\nu}^{\mu}(x)=\left(\frac{\delta L}{\delta g_{\alpha \beta}} g_{\alpha \beta \nu}\right)(x) .
$$

But $g_{\alpha \beta \nu}(x)=0$ in normal coordinates.

The proof of this result does not rely on the field equations for the metric. (Indeed, $g$ may not even have field equations.) In particular, it is not necessary to couple a field theory to gravity and appeal to Einstein's equations to force $\mathcal{T}^{\mu}{ }_{\nu}$ to be covariantly conserved; Proposition $\mathbf{5}$ is a general property of $\mathcal{T}^{\mu}{ }_{\nu}$. See Fischer [1982, 1985] for further discussion of this and related matters.

We wish to mention some special situations. Suppose first of all that the matter fields are non-derivatively coupled to $g$. Then the metric contribution to $\mathcal{T}^{\mu}{ }_{\nu}$ (viz. the last two terms in (4.2)) disappears, and $\mathcal{T}^{\mu}{ }_{\nu}$ reduces to the "ordinary" matter SEM tensor density:

$$
\mathcal{T}^{\mu}{ }_{\nu}=L \delta^{\mu}{ }_{\nu}-L_{a}{ }^{\mu} v^{a}{ }_{\nu}+L_{a}{ }^{\mu} C^{a}{ }_{\nu}+D_{\rho}\left(L_{a}{ }^{\rho} C^{a \mu}{ }_{\nu}\right) .
$$

On the other hand, consider the case when the $\psi^{a}$ are tensor fields, so that

$$
\nabla_{\nu} \psi^{a}=\psi_{, \nu}^{a}+C_{\tau}^{a \rho} \Gamma_{\rho \nu}^{\tau} .
$$

Then a straightforward calculation shows that (4.2) reduces to

$$
\mathcal{T}^{\mu}{ }_{\nu}=L \delta^{\mu}{ }_{\nu}-L_{a}{ }^{\mu} \nabla_{\nu} \psi^{a}+L_{a}{ }^{\mu} C^{a}{ }_{\nu}+\nabla_{\rho} K_{\nu}^{\mu \rho},
$$

where

$$
K_{\nu}^{\mu \rho}=L_{a}{ }^{\rho} C_{\nu}^{a \mu}-2 \frac{\partial L}{\partial g_{\mu \beta \rho}} g_{\nu \beta} .
$$

This expression for $\mathcal{T}^{\mu}{ }_{\nu}$ generalizes the formula (1.3) of Belinfante [1940] and Rosenfeld [1940], which in their context appeared as the relation between the Hilbert and 
canonical SEM tensors for tensor (or spinor) fields minimally coupled to gravity. In such cases, we define $\mathcal{T}^{\mu}{ }_{\nu}$ using only the matter Lagrangian $\mathcal{L}_{m}, c f$. (3.6). Then Theorem 2 shows that $\mathcal{T}^{\mu}{ }_{\nu}$ so defined coincides with the Hilbert SEM tensor, which thereby acquires a straightforward physical interpretation à la Noether.

To conclude this section, we briefly indicate what happens when one includes a frame - as opposed to a metric - among the field variables. Let $e_{a}$ be an orthonormal frame field on $X$ with components $e^{a}{ }_{\mu}$, where

$$
g_{\mu \nu}=e^{a}{ }_{\mu} e^{b}{ }_{\nu} \eta_{a b}
$$

for some nonsingular matrix $\eta_{a b}$. Then, mimicking the analysis above, one finds analogously to (4.3) that

$$
\mathcal{T}^{\mu}{ }_{\nu}=\frac{\delta L}{\delta e^{a}{ }_{\mu}} e^{a}{ }_{\nu}
$$

It does not necessarily follow from this that $\mathcal{T}_{\mu \nu}=g_{\mu \rho} \mathcal{T}^{\rho}{ }_{\nu}$ is symmetric. When the $e^{a}{ }_{\mu}$ appear in the Lagrangian density only through the intermediaries $g_{\mu \nu}$, then it is known that $\mathcal{T}_{\mu \nu}$ is symmetric (Rosenfeld [1940]). In more general circumstances, however, this need not be the case; see Künzle [1986] for an example.

\section{$5 \quad$ Examples}

\section{Electromagnetism}

The Maxwell theory provides a simple, yet nontrivial example of the set up in $\S 4$. We consider electromagnetism on an arbitrary nondynamic spacetime, regarded as an ordinary 1-form field theory, and we treat the spacetime metric $g$ as an auxiliary variable. Then $Y=\Lambda^{1} X \times_{X}$ Lor $(X)$, the fiber product of the bundle of one forms on $X$ with the bundle of Lorentz metrics on $X$, and the Lagrangian density is

$$
L=-\frac{1}{4} F_{\alpha \beta} F^{\alpha \beta} \sqrt{-g},
$$

where $F_{\alpha \beta}=A_{\beta, \alpha}-A_{\alpha, \beta}$. Since $\partial L / \partial A_{\alpha \beta}=F^{\alpha \beta} \sqrt{-g}$, the canonical SEM tensor (1.1) is

$$
\mathfrak{t}^{\mu \nu}=-\left[\frac{1}{4} g^{\mu \nu} F_{\alpha \beta} F^{\alpha \beta}+g^{\nu \beta} F^{\alpha \mu} A_{\alpha, \beta}\right] \sqrt{-g} .
$$

It is clearly neither symmetric nor gauge-invariant. To "fix" it, one adds the term $g^{\nu \beta} F^{\alpha \mu} A_{\beta, \alpha} \sqrt{-g}$, thereby producing

$$
\mathfrak{T}^{\mu \nu}=-\left[\frac{1}{4} g^{\mu \nu} F_{\alpha \beta} F^{\alpha \beta}+g^{\nu \beta} F^{\alpha \mu} F_{\beta \alpha}\right] \sqrt{-g} .
$$


We now derive (5.1) using the methods of this paper. The gauge group for electromagnetism is $\mathcal{G}=\operatorname{Diff}(X) \subseteq C^{\infty}(X)$, where $\operatorname{Diff}(X)$ acts on $C^{\infty}(X)$ by pushforward. The group $\mathcal{G}$ acts on $Y$ by

$$
(\eta, \lambda)_{Y} \cdot A=\eta_{*} A+d \lambda .
$$

The standard embedding of $\operatorname{Diff}_{c}(X)$ into $\mathcal{G}$ is simply $\eta \mapsto(\eta, 0)$. However, to illustrate the independence of our results upon the choice of embedding, we instead choose the nonstandard one given as follows: fix $\lambda \in C^{\infty}(X)$ and define $\eta \mapsto$ $\left(\eta, \eta_{*} \lambda-\lambda\right)$. Then the induced action of $\operatorname{Diff}_{c}(X)$ on $Y$ is

$$
\eta_{Y} \cdot A=\eta_{*} A+d\left(\eta_{*} \lambda-\lambda\right)
$$

Then for $\xi \in \mathfrak{X}_{c}(X)$,

$$
\xi_{Y}=\xi^{\nu} \partial_{\nu}-\left(A_{\nu} \xi_{, \alpha}^{\nu}+\lambda_{, \nu} \xi_{, \alpha}^{\nu}+\lambda_{, \alpha \nu} \xi^{\nu}\right) \frac{\partial}{\partial A_{\alpha}}
$$

so (3.2) holds with

$$
C_{\alpha \nu}^{\rho}=-\left(A_{\nu}+\lambda_{, \nu}\right) \delta^{\rho}{ }_{\alpha} \quad \text { and } \quad C_{\alpha \nu}=-\lambda_{, \alpha \nu} .
$$

Thus $k=1$, but note that zeroth order derivative terms appear as well.

Since the metric is nonderivatively coupled to $A$, we may apply (4.4) to obtain

$$
\begin{aligned}
\mathcal{T}^{\mu}{ }_{\nu}= & -\left[\frac{1}{4} F_{\alpha \beta} F^{\alpha \beta} \delta^{\mu}{ }_{\nu}+F^{\alpha \mu} A_{\alpha, \nu}\right] \sqrt{-g} \\
& -F^{\alpha \mu} \lambda_{, \alpha \nu} \sqrt{-g}-D_{\alpha}\left(F^{\mu \alpha}\left[A_{\nu}+\lambda_{, \nu}\right] \sqrt{-g}\right) .
\end{aligned}
$$

Using Maxwell's equations $D_{\alpha}\left(F^{\mu \alpha} \sqrt{-g}\right)=0$, the last term becomes

$$
-F^{\mu \alpha}\left(A_{\nu, \alpha}+\lambda_{, \nu \alpha}\right) \sqrt{-g}
$$

Substituting into the above and raising the index we obtain (5.1). All trace of $\lambda$ has disappeared, as it must by the general theory presented.

\section{Bosonic Strings}

Here we consider the Polyakov version of the bosonic string, $c f$. Green, Schwarz and Witten [1987]. Let $(X, h)$ be a 2-dimensional Lorentzian space, and $(M, g)$ the physical 4-dimensional spacetime. A bosonic string is a map $\psi: X \rightarrow M$, regarded as a (spacetime-valued) scalar field on $X$. In this formulation, the physical metric $g$ is fixed while the metric $h$ is dynamic. ${ }^{11}$ The Lagrangian density is

$$
L=-\frac{1}{2} \sqrt{|h|} h^{\sigma \rho} g_{A B} v_{\sigma}^{A} v_{\rho}^{B} .
$$

\footnotetext{
${ }^{11}$ In this regard we emphasize that, insofar as the considerations of $\S \mathbf{4}$ are concerned, it is the metric $h$ on $X$ that is relevant; the metric on $M$ is fixed and plays no essential role.
} 
We take the action of $\operatorname{Diff}_{c}(X)$ on the fields $(\psi, h)$ to be $(\eta,(\psi, h)) \mapsto\left(\eta_{*} \psi, \eta_{*} h\right)$. Then, since $h$ is nonderivatively coupled to $\psi,(4.4)$ gives

$$
\mathcal{T}_{\mu \nu}=\left[g_{A B} v_{\mu}^{A} v^{B}{ }_{\nu}-\frac{1}{2} h_{\mu \nu} h^{\alpha \beta} g_{A B} v_{\alpha}^{A} v^{B}{ }_{\beta}\right] \sqrt{|h|} .
$$

As $\psi$ is a scalar field, this coincides with the canonical SEM tensor.

One can also check directly that this is consistent with Theorem $\mathbf{2}$ :

$$
\mathcal{T}_{\mu \nu}=-2 \frac{\delta L}{\delta h^{\mu \nu}}
$$

But $h$ is dynamic, so $\mathcal{T}_{\mu \nu}$ vanishes on shell. This is expected in view of the form of the string Lagrangian, which - unlike the case of matter fields coupled to gravity — cannot be split into "matter" and "metric" parts à la (3.5).

In the jargon of elasticity theory, our treatment of the string corresponds to the "body" representation. This is reflected by the fact that our SEM tensor $\mathcal{T}_{\mu \nu}$ is a $2 \times 2$ matrix; in some sense it describes the "internal" distribution of energy, momentum and stress. Physically, then, the vanishing of $\mathcal{T}_{\mu \nu}$ on shell can be interpreted to mean that if the string is to be harmonically mapped into spacetime, then it must be "internally unstressed." To obtain the "physical" $4 \times 4$ SEM tensor on the spacetime $(M, g)$, one would have to work in the inverse material representation and consider a "cloud" of strings.

In this example, the parameter space $X$ was not the physical spacetime. This happens in other contexts as well. For instance, Künzle and Duval [1986] have constructed a Kaluza-Klein version of classical field theory; in their approach $X$ is a certain circle bundle over spacetime. The SEM tensor is then defined on this space and so is a $5 \times 5$ matrix; in the case of an adiabatic fluid, the additional components of $\mathcal{T}^{\mu}{ }_{\nu}$ can be interpreted as an entropy-flux vector (Künzle [1986]).

\section{Gravity}

Consider first the case of vacuum gravity. Then one has $\mathcal{T}^{\mu}{ }_{\nu}=-\frac{1}{8 \pi} \mathfrak{G}^{\mu}{ }_{\nu} \approx 0$. Recalling that in this instance $\mathcal{T}^{\mu}{ }_{\nu}$ measures the response of the gravitational field to localized spacetime deformations, one can interpret this result as saying that the energy and momentum of a vacuum gravitational field cannot be localized.

Now suppose matter is present. Then the analysis in $\S \mathbf{3}$ yields

$$
\mathcal{T}^{\mu}{ }_{\nu}=-\frac{1}{8 \pi} \mathfrak{G}^{\mu}{ }_{\nu}+\mathfrak{T}^{\mu}{ }_{\nu} \approx 0
$$

This can be thought of as expressing the balance of localizable energy and momentum of the total system. In particular, this equation leads one to ascribe to the gravitational field a certain tensorial stress-energy-momentum density in the amount of $-\frac{1}{8 \pi} \mathfrak{G}^{\mu}{ }_{\nu}$. Of course, this does not imply that all of the energy and momentum of the gravitational field is localizable. 
By its very constuction, $\mathcal{T}^{\mu}{ }_{\nu}$ is correlated with localizable energy and momentum. But not so the multimomentum map $J^{\mathcal{L}}$; in fact, its integral over a Cauchy surface $\Sigma$ should provide a measure of the total energy and momentum contained in $\Sigma$, localizable or not ( $c f$. Remark $\mathbf{5}$ ). To illustrate this point, consider an asymptotically flat gravitational field, in which context $\mathcal{D}$ (defined in Remark $\mathbf{5}$ above) consists of those diffeomorphisms of spacetime that are Poincaré at spatial infinity, cf. Ashtekar, Bombelli and Reula [1991]. Then for $\xi$ in the Lie algebra of $\mathcal{D}$, one finds that the left hand side of (3.5) reduces to a surface integral which may well represent the total energy and momentum of the gravitational field. Although we will not pursue this line of inquiry here, it is plausible that the formalism we have developed based upon the multimomentum map will provide a systematic means of studying questions concerning mass and angular momentum in general relativity.

\section{Topological Field Theory}

A "topological field theory" is, broadly speaking, a field theory in which there is no metric on the parameter space $X$. We use the abelian Chern-Simons theory to briefly illustrate our results in this context. Let $X$ be a 3 -manifold and take $Y=\Lambda^{1} X$. The Chern-Simons Lagrangian density is given by

$$
\mathcal{L}=\mathbf{d} A \wedge A,
$$

and the Euler-Lagrange equations are $F=\mathbf{d} A=0$. The gauge group is $\operatorname{Diff}(X)$ acting on $\Lambda^{1} X$ by push-forward. We compute

$$
\mathcal{T}^{\mu}{ }_{\nu}=\epsilon^{\mu \alpha \beta} A_{\nu} F_{\alpha \beta} .
$$

Once again $\mathcal{T}^{\mu}{ }_{\nu}$ vanishes on shell. This time, however, the interpretation is somewhat different: it reflects the fact that topological field theories have no "local physics," and hence no localizable "energy" or "momentum." (It is necessary to qualify these terms since, in the absence of a metric, one cannot distinguish one from the other.) As in the previous example, this does not preclude the possibility that such a theory has a total nonzero energy/momentum content. In fact, using the formula in Remark 5, we can explicitly compute the "topological" energy/momentum at infinity:

$$
-\int_{\Sigma} i_{\Sigma}^{*}\left(j^{1} A\right)^{*} J^{\mathcal{L}}\left(\xi_{Y}\right)=\int_{\partial \Sigma}[\mathbf{i}(\xi) A] A
$$

for arbitrary $\xi \in \mathfrak{X}(X)$.

\section{Relativistic Elasticity}

Relativistic elasticity has, as its basic fields, the world tube of the elastic material and a Lorentz metric $g$ on the physical spacetime $M$. The world tube is viewed as a map $\Phi: \mathcal{B} \times \mathbb{R} \rightarrow M$, where $\mathcal{B}$ is a 3-dimensional reference region and $\mathbb{R}$ is the time axis. The matter Lagrangian density is a given constitutive function of the 
relativistic spatial version of the Cauchy-Green tensor, namely $g+u \otimes u$, where $u$ is the world velocity field of $\Phi$. See Marsden and Hughes [1983], §5.7, for more details on how this is set up. Notice especially that we do not require that the spacetime be sliced with comoving coordinates or any other particular choice, following the lead of Bao, Marsden, and Walton [1985]. The spacetime diffeomorphism group acts on the world tube by composition on the left, which is a relativistic version of the principle of material frame indifference, and it acts on the metric as usual, by pushforward. Thus, our theory applies to this case, and so one must have the stress energy momentum tensor given by either via the Noether based definition (3.5), or equivalently via the Hilbert formula (4.3). The former seems not to be known in relativistic elasticity. The Hilbert formula is common in the literature and is found in Marsden and Hughes [1983], page 313, which can also be consulted for additional references.

Acknowledgements We are grateful for helpful discussions with Arthur Fischer, Hans Künzle, John Pierce, and Abe Taub.

\section{References}

Anderson, I.M. [1992] Introduction to the variational bicomplex. These proceedings.

Ashtekar, A., L. Bombelli and O. Reula [1991] The covariant phase space of asymptotically flat gravitational fields. In Mechanics, Analysis and Geometry: 200 Years After Lagrange, M. Francaviglia, ed., North Holland, Amsterdam, 417450.

Bao, D., J.E. Marsden and R. Walton [1984] The Hamiltonian structure of general relativistic perfect fluids. Comm. Math. Phys. 99, 319-345.

Belinfante, F.J. [1939] On the spin angular momentum of mesons. Physica vi, 887-898.

Belinfante, F.J. [1940] On the current and the density of the electric charge, the energy, the linear momentum and the angular momentum of arbitrary fields. Physica vii, 449-474.

Corson, E.M. [1953] Introduction to Tensors, Spinors and Relativistic Wave Equations. Hafner, New York.

Davis, W.R. [1970] Classical Fields, Particles, and the Theory of Relativity. Gordon and Breach, New York.

Epstein, D. and W. Thurston [1979] Transformation groups and natural bundles. Proc. Lon. Math. Soc. 38, 219-236. 
Ferraris, M. and M. Francaviglia [1983] A constructive approach to bundles of geometric objects on a differentiable manifold. J. Math. Phys. 24, 120-124.

Ferraris, M. and M. Francaviglia [1985] Energy-momentum tensors and stress tensors in geometric field theories. J. Math. Phys. 26, 1243-1252.

Ferraris, M. and M. Francaviglia [1991] The Lagrangian approach to conserved quantities in general relativity. In Mechanics, Analysis, and Geometry: 200 Years After Lagrange, M. Francaviglia, ed., North Holland, Amsterdam, 451488.

Fischer, A.E. [1982] A unified approach to conservation laws in general relativity, gauge theories, and elementary particle physics. Gen. Rel. and Grav. 14, 683-689.

Fischer, A.E. [1985] Conservation laws in gauge field theories. In Differential Geometry, Calculus of Variations, and their Applications, G.M. Rassias and T.M. Rassias, eds., Marcel Dekker, N.Y., 211-253.

Gotay, M.J., J.A. Isenberg, J.E. Marsden and R. Montgomery [1992] Momentum Maps and Classical Relativistic Fields (in preparation).

Green, M.B., J.H. Schwarz, and E. Witten [1987] Superstring Theory I. Introduction. Cambridge University Press.

Hawking, S.W. and G.F.R. Ellis [1973] The Large Scale Structure of Space-time. Cambridge University Press.

Kijowski, J. and W. Tulczyjew [1979] A Symplectic Framework for Field Theories. Springer Lect. Notes in Physics 107.

Kuchař, K. [1973] Canonical quantization of gravity. In Relativity, Astrophysics and Cosmology, W. Israel, ed., Reidel, Dordrecht, 237-288.

Künzle, H.P. [1986] Lagrangian formalism for adiabatic fluids on five-dimensional spacetime. Can. J. Phys. 64, 185-189.

Künzle, H.P. and C. Duval [1986] Relativistic and non-relativistic classical field theory on five-dimensional spacetime. Class. Quantum Grav. 3, 957-973.

Love, A.E.H [1944] A Treatise on the Mathematical Theory of Elasticity. Dover, New York.

Marsden, J.E. and T.J.R. Hughes [1983] Mathematical Foundations of Elasticity. Prentice-Hall, Redwood City, CA.

Misner, C.W., K. Thorne and J.A. Wheeler [1973] Gravitation. W. H. Freeman, San Francisco.

Palais, R. and C.-L. Terng [1977] Natural bundles have finite order. Topology 16, 271-277. 
Rosenfeld, L. [1940] Sur le tenseur d'impulsion-énergie. Mém. Acad. Roy. Belg. Sci. 18, 1-30.

Simo, J.C. and J.E. Marsden [1984] On the rotated stress tensor and a material version of the Doyle-Ericksen formula. Arch. Rat. Mech. An. 86, 213-231.

Souriau, J.-M. [1974] Modèle de particule à spin dans le champ éléctromagnétique et gravitationnel. Ann. Inst. H. Poincaré 20, 315-364.

Taub, A.H. [1970] Variational principles in general relativity. CIME lectures, Bressanone.

Trautman, A. [1965] Foundations and current problems of general relativity. In Lectures on General Relativity. Vol. I, Prentice-Hall, Englewood Cliffs, NJ, $1-248$.

Wentzel, G. [1949] Quantum Theory of Fields. Interscience, New York. 